\title{
UNCERTAINTY PROPAGATION FOR TERRESTRIAL MOBILE LASER SCANNER
}

\author{
Miloud Mezian*, Bruno Vallet *, Bahman Soheilian*, Nicolas Paparoditis* \\ * Universite Paris-Est, IGN, SRIG, MATIS, 73 avenue de Paris, 94160 Saint Mande, France
}

(firstname.lastname@ign.fr)

Commission III, WG III/2

KEY WORDS: Mobile mapping, Laser scanner, Uncertainty propagation, Error ellipsoid

\begin{abstract}
:
Laser scanners are used more and more in mobile mapping systems. They provide 3D point clouds that are used for object reconstruction and registration of the system. For both of those applications, uncertainty analysis of 3D points is of great interest but rarely investigated in the literature. In this paper we present a complete pipeline that takes into account all the sources of uncertainties and allows to compute a covariance matrix per $3 \mathrm{D}$ point. The sources of uncertainties are laser scanner, calibration of the scanner in relation to the vehicle and direct georeferencing system. We suppose that all the uncertainties follow the Gaussian law. The variances of the laser scanner measurements (two angles and one distance) are usually evaluated by the constructors. This is also the case for integrated direct georeferencing devices. Residuals of the calibration process were used to estimate the covariance matrix of the 6D transformation between scanner laser and the vehicle system. Knowing the variances of all sources of uncertainties, we applied uncertainty propagation technique to compute the variance-covariance matrix of every obtained 3D point. Such an uncertainty analysis enables to estimate the impact of different laser scanners and georeferencing devices on the quality of obtained 3D points. The obtained uncertainty values were illustrated using error ellipsoids on different datasets.
\end{abstract}

\section{INTRODUCTION}

Mobile Terrestrial LiDAR System (MTLS) is an emerging technology that combines the use of a laser scanner, Global Navigation System (GPS), Inertial Navigation System (INS) and odometer on a mobile platform to produce accurate and precise geospatial data on canyon urban. Much more detailed information has been acquired in comparaison with Airborne LiDAR Systems (ALS). (Olsen, 2013) discussed the key differences and similarties between airborne and mobile LiDAR data. The MTLS allows to obtain a large amount of $3 \mathrm{D}$ positional information in a fast and efficient way, which can be used in numerous applications, such as the object reconstruction and registration of system. In such applications, uncertainty analysis of $3 \mathrm{D}$ points is of great interest but rarely investigated in the literature. Consequently, it is important for users to know the uncertainty of MTLS and the factors that can influence the quality of 3D scanned data. Several studies have analysed the sources of uncertainties in MTLS, which are similar to those used by Airborne LiDAR System (ALS). More detail about ALS uncertainty sources was introduced by (Schaer et al., 2007). Others issues, related to sources of uncertainty affecting on the accuracy of the Mobile Terrestrial LiDAR point cloud are discussed in (Alshawa et al., 2007, Olsen, 2013, Leslar et al., 2014, Poreba, 2014) .

In general, the sources of uncertainty are divided into three main categories:

- navigation uncertainties : Here we have uncertainty of the absolute position and the vehicule orientation measured by INS in real time. The factors that affect the accuracy of the vehicule's position depend : multipath, shading of the signals caused by buildings and trees, and poor GPS satellite geometry (Glennie, 2007, Haala et al., 2008). Under good GPS conditions this uncertainty is about few centimeters, however, under difficult conditions it can be up to few meters. In (Leslar et al., 2014), they have proved that under tightly controlled error conditions, the source of uncertainty in point cloud is domined by vehicule position.

- calibration uncertainties : Namely the uncertainties in the leverarm and in the boresight angles between scanner laser and the INS frame. The quality of the calibration parameters is usually known and depends on the calibration procedure (Le Scouarnec et al., 2014, Rieger et al., 2010).

- laser scanning uncertainties : The scanner laser measurements consist of two angulars and one distance.The factors affecting laser-target position accuracy are numerous such as the weather (humidity, temperature), properties of the scanned surface (roughness, reflectivity), scanning geometry (incidence angle on the surface) and scanner mechanism precision (mirror center offset) (Soudarissanane et al., 2008). These uncertainties are evaluated by the constructors.

This paper is organized as follows: Section 1 presents briefly the principles of geo-referencing of 3D point. Section 2 describes a complete pipeline that takes into account all the sources of uncertainties and allows to compute a covariance matrix per 3D point. The obtained uncertainty values were illustrated using error ellipsoids on different datasets.

In this work, the mobile data that we used was produced by the terrestrial mobile mapping vehicle Stereopolis II (Paparoditis et al., 2012) developped at National Geographic Institute (IGN). The laser sensor is a RIEGL VQ-250 that was mounted transversally in order to scan a plane orthogonal to the trajectory. It rotates at $100 \mathrm{~Hz}$ and emits 3000 pulses per rotation, which corresponds to an angular resolution around $0.12^{\circ}$ result in 0 to 8 echoes producing an average of 250 thousand points per second. 


\subsection{Mobile Terrestrial LiDAR formulas}

Calculation of ground coordinates from Mobile Terrestrial Lidar System (MTLS) have been well documented in the literature (Ellum and El-Sheimy, 2002). As shown in Figure (1), the coordinates of point $\mathbf{P}_{i}^{\mathrm{w}}$ in the World frame can be calculated by combining the information from the LiDAR, INS measurements and calibration

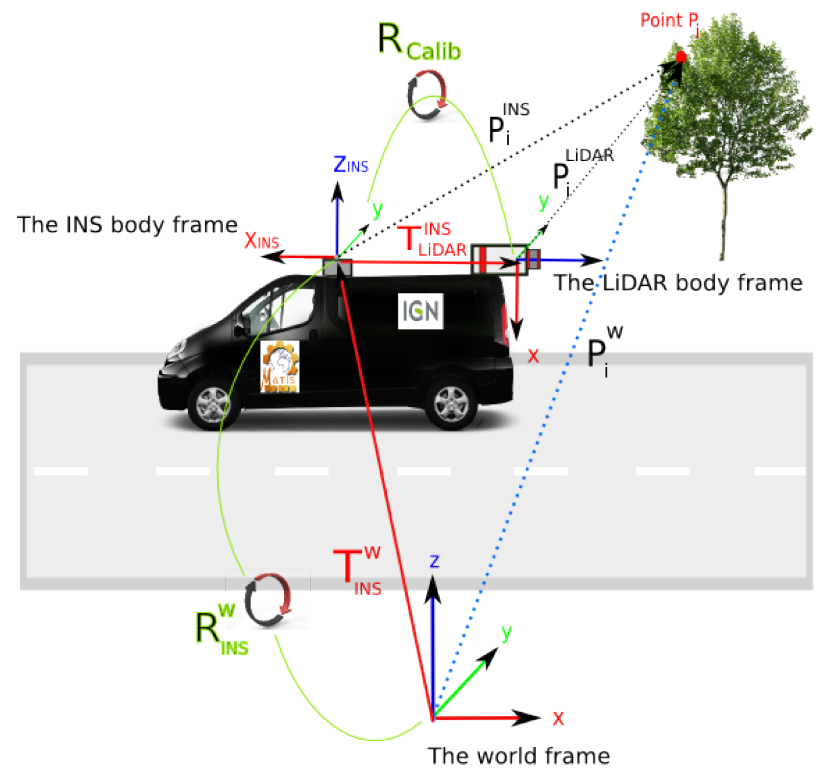

Figure 1: Concept of Mobile Terrestrial LiDAR System (MTLS)

The formula used to calculate the LiDAR point in the world frame is given in Equation (1) :

$$
\mathbf{P}_{i}^{\mathbf{W}}(t)=\mathbf{R}_{\mathbf{I N S}}^{\mathbf{W}}(t) \mathbf{P}_{i}^{\mathbf{I N S}}(t)+\mathbf{T}_{\mathbf{I N S}}^{\mathbf{W}}(t)
$$

Where :

- $\mathbf{P}_{i}^{\mathbf{w}}(t)$ represents the $3 \mathrm{D}$ coordinate of point $\mathrm{P}$ in the World frame at time $t$ and $i$ is the index of the point.

- $\mathbf{T}_{\mathbf{I N S}}^{\mathbf{W}}(t)$ is the position of the INS in the World frame at time $\mathrm{t}$.

- $\mathbf{R}_{\mathbf{I N S}}^{\mathbf{w}}(t)$ is the rotation matrix between the INS body frame and the World frame at time $t$ :

$$
\mathbf{R}_{\mathbf{I N S}}^{\mathbf{W}}(t)=\mathbf{R}_{\mathbf{N E D}}^{\mathbf{E N U}} \cdot \mathbf{R}\left(\theta_{\mathbf{z}}(t), \theta_{\mathbf{y}}(t), \theta_{\mathbf{x}}(t)\right)
$$

- $\mathbf{R}_{\mathbf{N E D}}^{\mathbf{E N U}}$ is the constant rotation matrix between the NorthEast-Down coordinates and the East-North-Up coordinate frame:

$$
\mathbf{R}_{\mathbf{N E D}}^{\mathbf{E N U}}=\left[\begin{array}{ccc}
0 & 1 & 0 \\
1 & 0 & 0 \\
0 & 0 & -1
\end{array}\right]
$$

- $\mathbf{R}\left(\theta_{\mathbf{z}}, \theta_{\mathbf{y}}, \theta_{\mathbf{x}}\right)$ is the rotation matrix between the INS body frame and the World frame (in the North- EastDown coordinate system) with $\theta_{\mathbf{x}}, \theta_{\mathbf{y}}, \theta_{\mathbf{z}}$ which represent the roll, pitch and yaw Euler angles given by the INS at time $t$ :

$$
\mathbf{R}\left(\theta_{\mathbf{z}}, \theta_{\mathbf{y}}, \theta_{\mathbf{x}}\right)=\mathbf{R}\left(\theta_{\mathbf{z}}\right) \mathbf{R}\left(\theta_{\mathbf{y}}\right) \mathbf{R}\left(\theta_{\mathbf{x}}\right)
$$

with :

$$
\begin{aligned}
\mathbf{R}\left(\theta_{\mathbf{z}}\right) & =\left[\begin{array}{ccc}
\cos \left(\theta_{\mathbf{z}}\right) & -\sin \left(\theta_{\mathbf{z}}\right) & 0 \\
\sin \left(\theta_{\mathbf{z}}\right) & \cos \left(\theta_{\mathbf{z}}\right) & 0 \\
0 & 0 & 1
\end{array}\right] \\
\mathbf{R}\left(\theta_{\mathbf{y}}\right) & =\left[\begin{array}{ccc}
\cos \left(\theta_{\mathbf{y}}\right) & 0 & \sin \left(\theta_{\mathbf{y}}\right) \\
0 & 1 & 0 \\
-\sin \left(\theta_{\mathbf{y}}\right) & 0 & \cos \left(\theta_{\mathbf{y}}\right)
\end{array}\right] \\
\mathbf{R}\left(\theta_{\mathbf{x}}\right) & =\left[\begin{array}{ccc}
1 & 0 & 0 \\
0 & \cos \left(\theta_{\mathbf{x}}\right) & -\sin \left(\theta_{\mathbf{x}}\right) \\
0 & \sin \left(\theta_{\mathbf{x}}\right) & \cos \left(\theta_{\mathbf{x}}\right)
\end{array}\right]
\end{aligned}
$$

- $\mathbf{P}_{i}^{\mathbf{I N S}}(t)$ is the position of the target point in the INS body frame at time $\mathrm{t}$ :

$$
\mathbf{P}_{i}^{\mathbf{I N S}}(t)=\mathbf{R}_{\text {calib }}(\Omega, \varphi, \kappa) \mathbf{P}_{i}^{\mathbf{L i D A R}}(t)+\mathbf{T}_{\mathbf{L i D A R}}^{\mathbf{I N S}}
$$

- $\mathbf{R}_{\text {calib }}(\Omega, \varphi, \kappa)$ is the constant rotation matrix between the LiDAR body frame and the INS body frame. $\kappa, \varphi$ and $\Omega$ are boresight angles.

- $\mathbf{T}_{\mathbf{L i D A R}}^{\mathrm{INS}}$ is the lever arm vector from the INS body frame to the LiDAR body frame.

- $\mathbf{P}_{i}^{\mathrm{LiDAR}}(t)$ is the laser range vector between the LiDAR and the target point $\mathrm{p}$ at time $\mathrm{t}$ :

$$
\mathbf{P}_{i}^{\mathbf{L i D A R}}(t)=\overbrace{\left[\begin{array}{c}
X_{0}^{\mathbf{L i D A R}}(t) \\
Y_{0}^{\mathbf{L i D A R}}(t) \\
Z_{0}^{\mathbf{L i D A R}}(t)
\end{array}\right]}^{\mathbf{P}_{\mathbf{L}}^{\mathbf{L i D A R}}(t)}+\left[\begin{array}{c}
\rho(t) \cos (\theta(t)) \sin (\phi(t)) \\
\rho(t) \sin (\theta(t)) \sin (\phi(t)) \\
\rho(t) \cos (\phi(t))
\end{array}\right]
$$

$\mathbf{P}_{\mathbf{0}}^{\mathrm{LiDAR}}(t)$ : represents the mirror center offset of the LiDAR instrument at time $\mathrm{t}$ (see Figure 2).

$\rho(t) \quad:$ is the LiDAR range at time $\mathrm{t}$ (see Figure 2).

$\theta(t), \phi(t) \quad$ : represents respectively the horizontal and vertical angles measured by the LiDAR in the LiDAR body frame at time $t$ (see Figure 2).
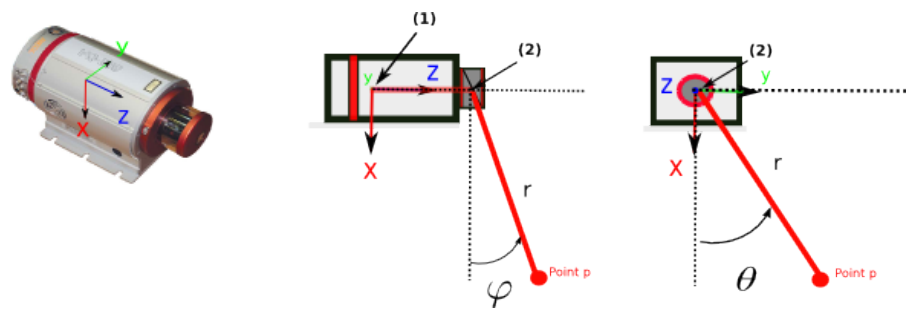

Figure 2: (1) Center of LiDAR frame - (2) The mirror center offset of the LiDAR instrument $\mathbf{P}_{\mathbf{0}}^{\mathrm{LiDAR}}$ 
The equation (1) can be rewritten as :

$$
\begin{gathered}
{\left[\begin{array}{c}
X_{i}^{\mathbf{w}}(t) \\
Y_{i}^{\mathbf{w}}(t) \\
Z_{i}^{\mathbf{w}}(t)
\end{array}\right]=\mathbf{R}_{\mathbf{N E D}}^{\mathbf{E N U}} \mathbf{R}\left(\theta_{\mathbf{z}}(t), \theta_{\mathbf{y}}(t), \theta_{\mathbf{x}}(t)\right) .} \\
{\left[\mathbf{R}_{\mathbf{c a l i b}}(\Omega, \varphi, \kappa) .\right.} \\
\left.\left[\begin{array}{c}
X_{0}^{\mathbf{L i D A R}}(t) \\
Y_{0}^{\mathbf{L i D A R}}(t) \\
Z_{0}^{\mathbf{L i D A R}}(t)
\end{array}\right]+\left[\begin{array}{c}
\rho(t) \cos (\theta(t)) \sin (\phi(t)) \\
\rho(t) \sin (\theta(t)) \sin (\phi(t)) \\
\rho(t) \cos (\phi(t))
\end{array}\right]\right]+\left[\begin{array}{l}
\mathbf{T}_{\mathbf{L i D A R}}^{\mathbf{I N S}} \\
\mathbf{T}_{\mathbf{L i D A R}}^{\mathbf{I N S}} \\
\mathbf{T}_{\mathbf{L i D A R}}^{\mathbf{I N S}}
\end{array}\right] \\
+ \\
+\left[\begin{array}{l}
\mathbf{T}_{\mathbf{I N S}}^{\mathbf{W}}(t) \\
\mathbf{T}_{\mathbf{I N S}}^{\mathbf{W}}(t) \\
\mathbf{T}_{\mathbf{I N S}}^{\mathbf{W}}(t)
\end{array}\right]
\end{gathered}
$$

The above equation shows that the coordinates of point $\mathbf{P}_{i}^{\mathbf{w}}$ in the World frame depends on 18 observations :

- $\mathbf{T}_{\mathbf{I N S}}^{\mathbf{W}}, \mathbf{T}_{\mathbf{I N S}}^{\mathbf{W}}$ and $\mathbf{T}_{\mathbf{I N S}}^{\mathbf{W}}$ defining the position of the INS in the World frame.

- $\theta_{\mathbf{x}}, \theta_{\mathbf{y}}$ and $\theta_{\mathbf{z}}$ are the roll, pitch and yaw Euler angles given by the INS.

The MTLS use an odometer, GPS, and INS to determine the position $\left(\mathbf{T}_{\mathbf{I N S}}^{\mathbf{W}}, \mathbf{T}_{\mathbf{I N S}}^{\mathbf{W}}, \mathbf{T}_{\mathbf{I N S}}^{\mathbf{W}}\right)$ and orientation $\left(\theta_{\mathbf{x}}, \theta_{\mathbf{y}}, \theta_{\mathbf{z}}\right)$ of vehicule at time t.

- $\mathbf{T}_{\mathbf{L i D A R}_{x}}^{\mathrm{INS}}, \mathbf{T}_{\mathbf{L i D A R}_{y}}^{\mathrm{INS}}$ and $\mathbf{T}_{\mathbf{L i D A R}_{z}}^{\mathrm{INS}}$ are the lever arm offsets from the INS body frame to the LiDAR body frame.

- $\Omega, \varphi, \kappa$ are the boresight angles which align the LiDAR body frame with the INS body frame.

The lever-arm offset $\left(\mathbf{T}_{\mathbf{L i D A R}_{x}}^{\mathrm{INS}}, \mathbf{T}_{\mathbf{L i D A R}}^{\mathrm{INS}}\right.$ and $\left.\mathbf{T}_{\mathbf{L i D A R}}^{\mathrm{INS}}\right)$ and boresight angles $\Omega, \varphi, \kappa$ are determined through a calibration procedure.

- $X_{0}^{\mathrm{LiDAR}}, Y_{0}^{\mathrm{LiDAR}}$ and $Z_{0}^{\mathrm{LiDAR}}$ represent the mirror center offsets of the LiDAR instrument given by the LiDAR scanner.

- $\rho, \theta$ and $\phi$ represent the range and scan angles measured given by the LiDAR scanner.

Each of the 18 observations have an uncertainty, in the form of an standard deviation denoted by $\sigma$. We suppose that each of the measurements follows the normal (Gaussian) distribution. Knowing the standart deviations $\sigma$ of all observations (see Table 1), we applied uncertainty propagation technique to compute the covariance matrix of every obtained 3D point.

\section{UNCERTAINTY PROPAGATION}

In this section, we present uncertainty propagation technique which is based on the Gauss-Helmert general method. By applying this method, it is possible to compute the covariance matrix of a $3 \mathrm{D}$ point. The objective is to convert the covariance matrix given in terms of observations to a covariance matrix in terms of parameters.

\subsection{General Methodology}

The generic form of the Gauss-Helmert method presented by (Vanicek and Krakiwsky, 2015) consists in resolution of the following equation system :

$$
\mathbf{F}(\hat{\boldsymbol{X}}, \hat{\boldsymbol{l}})=\mathbf{0}
$$

where $\hat{\boldsymbol{X}}$ is estimated unknown vector and $\hat{\boldsymbol{l}}$ is estimated observation vector. The linearized form of equation (10) is given in equation (11) :

$$
\mathbf{A} \hat{\boldsymbol{\delta}}+\mathbf{B} \hat{\boldsymbol{r}}+\mathbf{w}=0
$$

where $\mathbf{A}=\left.\frac{\partial \mathbf{F}}{\partial \mathbf{X}}\right|_{\mathbf{X}^{(0)}, \mathbf{l}^{(0)}}$ is the matrix of partial derivatives with respect to unknowns, $\mathbf{B}=\left.\frac{\partial \mathbf{F}}{\partial \mathbf{l}}\right|_{\mathbf{X}^{(\boldsymbol{\theta})}, \mathbf{l}^{(0)}}$ is the matrix of partial derivatives with respect to observations, $\mathbf{w}=\mathbf{F}\left(\mathbf{X}^{(\mathbf{0})}, \mathbf{l}^{(\mathbf{0})}\right)$ is the misclosure vector, $\mathbf{X}^{(\mathbf{0})}$ and $\mathbf{l}^{(\mathbf{0})}$ are intial values, $\hat{\boldsymbol{\delta}}$ and $\hat{\boldsymbol{r}}$ are residuals of unknowns and observations. The quantities $\mathbf{A}, \mathbf{B}$ and $\mathbf{w}$ are known, while $\hat{\boldsymbol{\delta}}$ and $\hat{\boldsymbol{r}}$ are unknown. The unknowns correction vector $\hat{\boldsymbol{\delta}}$ and observations correction vector $\hat{\boldsymbol{r}}$ are :

$$
\begin{aligned}
& \hat{\boldsymbol{\delta}}=-\left(\mathbf{A}^{\mathbf{T}} \mathbf{M} \mathbf{A}\right)^{-1} \mathbf{A}^{\mathbf{T}} \mathbf{M w} \\
& \hat{\boldsymbol{r}}=-\mathbf{C}_{\mathbf{r}} \mathbf{B}^{\mathbf{T}} \mathbf{M}(\mathbf{A} \hat{\boldsymbol{\delta}}+\mathbf{w})
\end{aligned}
$$

where $\mathbf{M}=\left(\mathbf{B C}_{\mathbf{r}} \mathbf{B}^{\mathbf{T}}\right)^{-1}$ and $\mathbf{C}_{\mathbf{r}}$ is the covariance matrix of the observations.

Finally, the general law of propagation of variances is applied. And, we obtain the covariance matrix of parameters :

$$
\mathbf{C}_{\mathbf{x}}=\left(\mathbf{A}^{\mathbf{T}} \mathbf{M A}\right)^{-1}
$$

\subsection{The covariance matrix of a $3 \mathrm{D}$ point}

The equation (1) can be rewritten in the generic form of the GaussHelmert method :

$$
\begin{aligned}
& \mathbf{F}\left(\hat{\boldsymbol{X}}_{i}, \hat{\boldsymbol{l}}_{i}\right)=\left[\begin{array}{c}
X_{i}^{\mathbf{w}}(t) \\
Y_{i}^{\mathbf{w}}(t) \\
Z_{i}^{\mathbf{w}}(t)
\end{array}\right]-\mathbf{R}_{\mathbf{N E D}}^{\mathbf{E N U}} \mathbf{R}\left(\theta_{\mathbf{z}}(t), \theta_{\mathbf{y}}(t), \theta_{\mathbf{x}}(t)\right) . \\
& {\left[\mathbf{R}_{\text {calib }}(\Omega, \varphi, \kappa) .\right.} \\
& \left.\left.\left[\begin{array}{c}
X_{0}^{\mathbf{L i D A R}}(t) \\
Y_{0}^{\mathbf{L i D A R}}(t) \\
Z_{0}^{\mathbf{L i D A R}}(t)
\end{array}\right]+\left[\begin{array}{c}
\rho(t) \cos (\theta(t)) \sin (\phi(t)) \\
\rho(t) \sin (\theta(t)) \sin (\phi(t)) \\
\rho(t) \cos (\phi(t))
\end{array}\right]\right]+\left[\begin{array}{l}
\mathbf{T}_{\mathbf{L i D A R}}^{\mathbf{I N S}} \\
\mathbf{T}_{\mathbf{L i D A R}}^{\mathbf{I N S}} \\
\mathbf{T}_{\mathbf{L i D A R}}^{\mathbf{I N S}}
\end{array}\right]\right] \\
& +\left[\begin{array}{l}
\mathbf{T}_{\mathbf{I N S}}^{\mathbf{W}}(t) \\
\mathbf{T}_{\mathbf{I N}}^{\mathbf{W}}(t) \\
\mathbf{T}_{\mathbf{I N \mathbf { S } _ { z }}}^{\mathbf{W}}(t)
\end{array}\right]=0
\end{aligned}
$$


Table 1: Sources of uncertainty in MTLS

\begin{tabular}{|c|c|c|c|}
\hline Sources of uncertainty & Observations & Uncertainties & Values \\
\hline Navigation uncertainties & $\begin{array}{c}\mathbf{T}_{\mathbf{I N S}}^{\mathbf{w}}(t), \text { Position X of the INS [m] } \\
\mathbf{T}_{\mathbf{I N S}}^{\mathbf{w}}(t) \text {, Position Y of the INS [m] } \\
\mathbf{T}_{\mathbf{I N S}}^{\mathbf{w}}(t) \text {, Position Z of the INS [m] } \\
\theta_{\mathbf{x}}(t) \text {, INS Roll [degrees] } \\
\theta_{\mathbf{y}}(t) \text {, INS Pitch [degrees] } \\
\theta_{\mathbf{z}}(t) \text {, INS Yaw [degrees] }\end{array}$ & $\begin{array}{c}\sigma_{\mathbf{T}_{\mathbf{N S}}^{\mathbf{w}}} \\
\sigma_{\mathbf{T}_{\mathbf{N S}}^{\mathbf{w}}}^{\mathbf{w}} \\
\sigma_{\mathbf{T}_{\mathbf{I N S}}^{\mathbf{w}}} \\
\sigma_{\theta_{\mathbf{x}}} \\
\sigma_{\theta_{\mathbf{y}}} \\
\sigma_{\theta_{\mathbf{z}}}\end{array}$ & $\begin{array}{l}\text { Estimated from INS } \\
\text { Estimated from INS } \\
\text { Estimated from INS } \\
\text { Estimated from INS } \\
\text { Estimated from INS } \\
\text { Estimated from INS }\end{array}$ \\
\hline Calibration uncertainties & 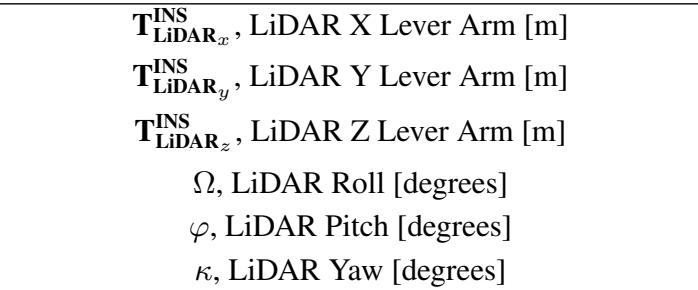 & 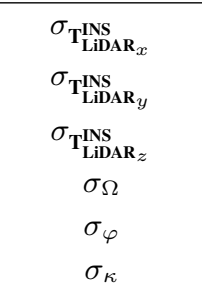 & $\begin{array}{c}0.001 \\
0.001 \\
0.001 \\
0.1 \\
0.1 \\
0.1\end{array}$ \\
\hline Laser scanning uncertainties & $\begin{array}{c}X_{0}^{\mathrm{LiDAR}}(t), \text { mirror center offset in the } \mathrm{X} \text { direction }[\mathrm{m}] \\
Y_{0}^{\mathrm{LiDAR}}(t), \text { mirror center offset in the } \mathrm{Y} \text { direction }[\mathrm{m}] \\
Z_{0}^{\mathrm{LiDAR}}(t), \text { mirror center offset in the } \mathrm{Z} \text { direction }[\mathrm{m}] \\
\quad \rho(t), \text { LiDAR Distance [m] } \\
\theta(t), \text { LiDAR Horizontal angle [degrees] } \\
\phi(t), \text { LiDAR Vertical angle [degrees] }\end{array}$ & $\begin{array}{c}\sigma_{X_{0}^{\mathrm{LiDAR}}} \\
\sigma_{Y_{0}^{\mathrm{LiDAR}}} \\
\sigma_{Z_{0}^{\mathrm{LiDAR}}} \\
\sigma_{\rho} \\
\sigma_{\theta} \\
\sigma_{\phi}\end{array}$ & $\begin{array}{c}0.001 \\
0.001 \\
0.001\end{array}$ \\
\hline
\end{tabular}

where :

- $\hat{\boldsymbol{X}}_{i}=\left[\begin{array}{c}X_{i}^{\mathbf{w}} \\ Y_{i}^{\mathbf{w}} \\ Z_{i}^{\mathbf{w}}\end{array}\right]$ : is the vector of unknowns of the $\mathbf{P}_{i}^{\mathbf{w}}$ point.

- $\hat{\boldsymbol{l}}_{i}=\left[\begin{array}{llllllll}\rho & \theta & \phi & X_{0}^{\mathrm{LiDAR}} & Y_{0}^{\mathrm{LiDAR}} Z_{0}^{\mathrm{LiDAR}} \Omega & \varphi & \kappa & \mathbf{T}_{\mathbf{L i D A R}_{x}}^{\mathrm{INS}}\end{array}\right.$ $\left.\begin{array}{llllllll}\mathbf{T}_{\mathbf{L i D A R}_{y}}^{\mathbf{I N S}} & \mathbf{T}_{\mathbf{L i D A R}_{z}}^{\mathbf{I N S}} & \theta_{\mathbf{x}} & \theta_{\mathbf{y}} & \theta_{\mathbf{z}} & \mathbf{T}_{\mathbf{I N S}}^{\mathbf{W}} & \mathbf{T}_{\mathbf{I N S}_{y}}^{\mathbf{W}} & \mathbf{T}_{\mathbf{I N S}}^{\mathbf{W}}\end{array}\right]^{\mathbf{T}}:$ is vector of observations of the $\mathbf{P}_{i}^{\mathbf{w}}$ point.

- $\mathbf{A}_{(3 \times 3)}=\mathbb{1}$ : is the matrix of partial derivatives with respect to unknown.

- $\mathbf{B}_{(3 \times 18)}=\left.\frac{\partial \mathbf{F}}{\partial \mathbf{l}}\right|_{\mathbf{l}^{(0)}}$ is the matrix of partial derivatives with respect to observations.

- $\mathbf{w}=\mathbf{F}\left(\mathbf{X}^{(\mathbf{0})}, \mathbf{l}^{(\mathbf{0})}\right)$ is the misclosure vector.

- $\hat{\boldsymbol{\delta}}=-\mathbf{w}, \hat{\boldsymbol{r}}=0$ are the unknowns correction vector and observations correction vector.

- $\mathbf{C}_{\mathbf{r}}^{i}$ is the covariance matrix of the observations of the $\mathbf{P}_{i}^{\mathbf{w}}$ point.

$$
\mathbf{C}_{\mathbf{r}}^{i}=\left[\begin{array}{cccc}
\sigma_{\rho}^{2} & 0 & \ldots & 0 \\
0 & \sigma_{\theta}^{2} & \ldots & 0 \\
\vdots & \vdots & \ddots & \vdots \\
0 & 0 & \ldots & \sigma_{\mathbf{T}_{\mathbf{N S}}^{\mathbf{w}} s_{z}}^{2}
\end{array}\right]
$$

We assume that the observations are independent, so all nondiagonal values in the matrix $\mathbf{C}_{\mathbf{r}}^{i}$ are equal to zero. The covariance matrix of $\mathbf{P}_{i}^{\mathrm{w}}$ can be computed by the covariance law :

$$
\mathbf{C}_{\mathbf{x}(3 \times 3)}^{i}=\mathbf{B C}_{\mathbf{r}} \mathbf{B}^{\mathbf{T}}=\left[\begin{array}{ccc}
\sigma_{x}^{2} & \sigma_{x y} & \sigma_{x z} \\
\sigma_{x y} & \sigma_{y}^{2} & \sigma_{y z} \\
\sigma_{x z} & \sigma_{y z} & \sigma_{z}^{2}
\end{array}\right]
$$

This covariance matrix of the point $\mathbf{P}_{i}^{\mathbf{w}}$ can be depicted by an error ellipsoid.

\subsection{Error ellipsoid}

From the covariance matrix of parameters (Equation 16) we can then calculate the eigenvalues $\left(\lambda_{1}>\lambda_{2}>\lambda_{3}\right)$ and eigenvectors $\left(\boldsymbol{e}_{1}, \boldsymbol{e}_{2}, \boldsymbol{e}_{3}\right)$. Each eigenvalue and eigenvector was used to construct the three axes of an ellipsoid. The eigenvectors give the directions of the principal axes of the uncertainty ellipsoid, and the eigenvalues give the variances along these principal axes. To create a $99.9 \%$ confidence ellipse from the $3 \sigma$ error, we must enlarge it by a factor of scale factor $s=\sqrt{11.345}$. The ellipsoid is centered on the point $\mathbf{P}_{i}^{\mathbf{w}}$ and the principal axes of this ellipse are determined by the following equations : $\mathbf{v}_{1}=s \lambda_{1} \mathbf{e}_{1}$, $\mathbf{v}_{2}=s \lambda_{1} \mathbf{e}_{2}$ and $\mathbf{v}_{3}=s \lambda_{1} \mathbf{e}_{3}$.

The error ellipsoids are illustrated in the figures (3), (4), (5) and (6) :

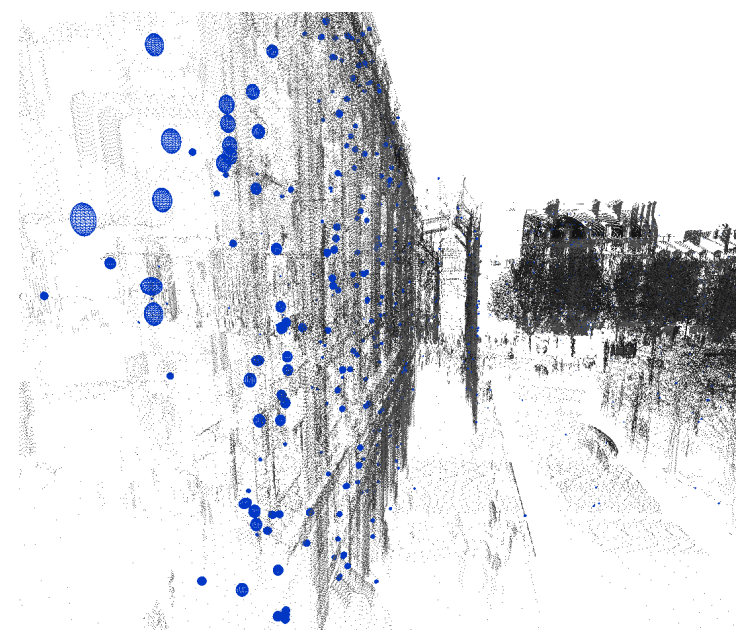

Figure 3: Black : points cloud of the mobile terrestrial LiDAR, blue : error ellipsoids. We estimate the error ellipsoid every 1000 points 


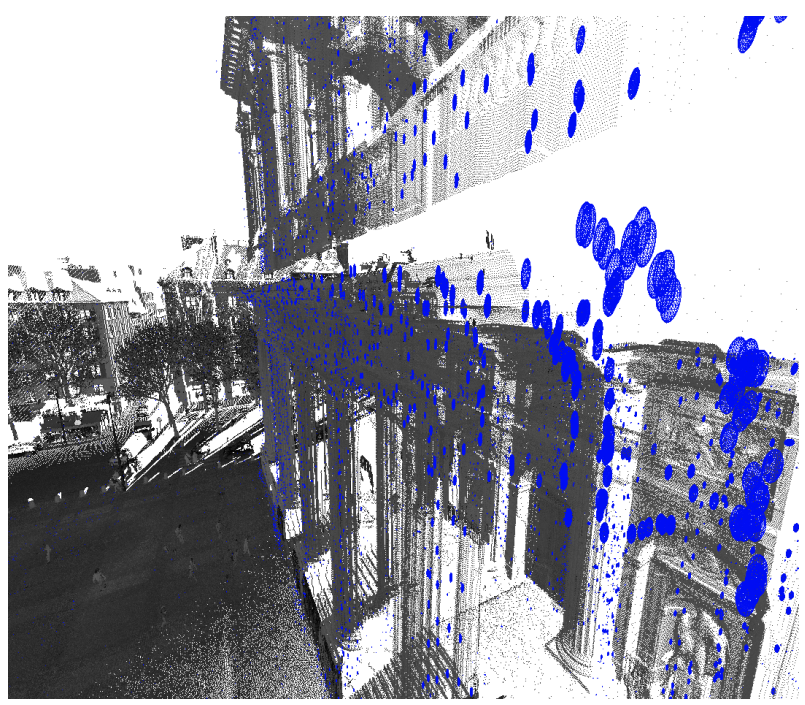

Figure 4: Black : points cloud of the mobile terrestrial LiDAR, blue : error ellipsoids. An error ellipsoid every 300 points

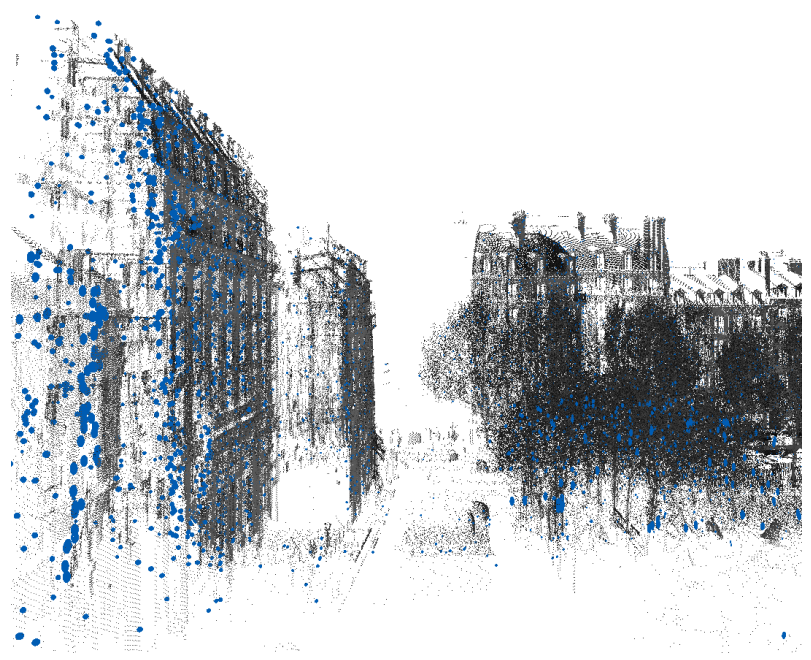

Figure 5: Black : points cloud of the mobile terrestrial LiDAR, blue : error ellipsoids. An error ellipsoid every 150 points

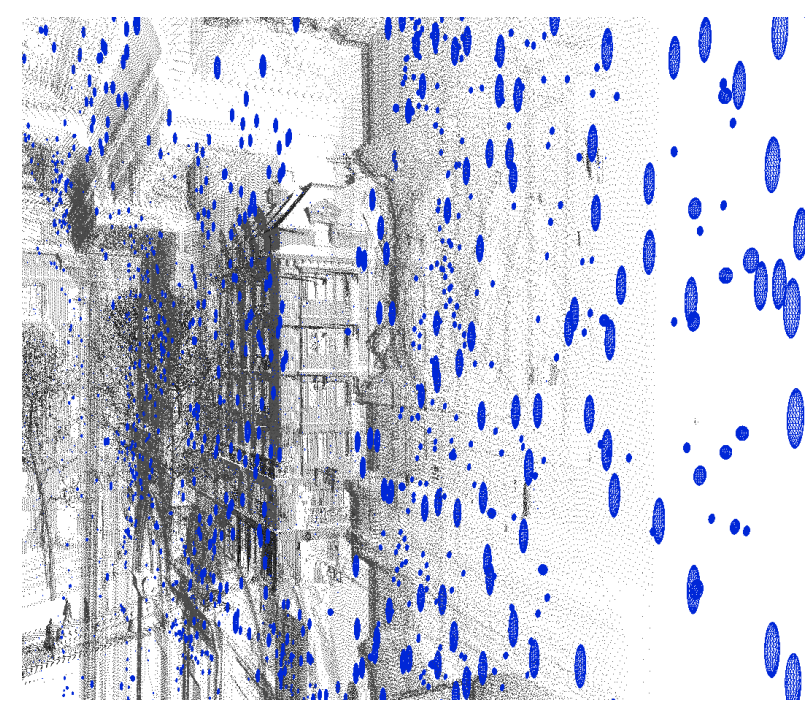

Figure 6: Black : points cloud of the mobile terrestrial LiDAR, blue : error ellipsoids. An error ellipsoid every 100 points

\section{CONCLUSION}

In this paper, we have presented a uncertainty propagation technique based on the general Gauss-Helmert method to compute the covariance matrix per 3D point and the obtained uncertainty values were illustrated using error ellipsoids on different datasets.

\section{REFERENCES}

Alshawa, M., Smigiel, E., Grussenmeyer, P. and Landes, T., 2007. Integration of a terrestrial lidar on a mobile mapping platformfirst experiences. In: International Symposium on Mobile Mapping Technology.

Ellum, C. and El-Sheimy, N., 2002. Land-based mobile mapping systems. Photogrammetric engineering and remote sensing 68(1), pp. 13-17.

Glennie, C., 2007. Rigorous 3d error analysis of kinematic scanning lidar systems. Journal of Applied Geodesy jag 1(3), pp. 147157.

Haala, N., Peter, M., Kremer, J. and Hunter, G., 2008. Mobile lidar mapping for $3 \mathrm{~d}$ point cloud collection in urban areas, a performance test. The international archives of the photogrammetry, remote sensing and spatial information sciences 37, pp. 11191127.

Le Scouarnec, R., Touzé, T., Lacambre, J.-B. and Seube, N., 2014. A new reliable boresight calibration method for mobile laser scanning applications. The International Archives of Photogrammetry, Remote Sensing and Spatial Information Sciences 40(3), pp. 67.

Leslar, M., Hu, B. and Wang, J., 2014. Error analysis of a mobile terrestrial lidar system. GEOMATICA 68(3), pp. 183-194.

Olsen, M. J., 2013. Guidelines for the use of mobile LIDAR in transportation applications. Vol. 748, Transportation Research Board.

Paparoditis, N., Papelard, J.-P., Cannelle, B., Devaux, A., Soheilian, B., David, N. and Houzay, E., 2012. Stereopolis ii: A multi-purpose and multi-sensor $3 \mathrm{~d}$ mobile mapping system for street visualisation and $3 \mathrm{~d}$ metrology. Revue française de photogrammétrie et de télédétection 200(1), pp. 69-79.

Poreba, M., 2014. Edge-based accuracy assessment and improvement of mobile laser scanning systems. Theses, Ecole Nationale Supérieure des Mines de Paris.

Rieger, P., Studnicka, N., Pfennigbauer, M. and Zach, G., 2010. Boresight alignment method for mobile laser scanning systems. Journal of Applied Geodesy 4(1), pp. 13-21.

Schaer, P., Skaloud, J., Landtwing, S. and Legat, K., 2007. Accuracy estimation for laser point cloud including scanning geometry. In: Mobile Mapping Symposium 2007, Padova.

Soudarissanane, S., Lindenbergh, R. and Gorte, B., 2008. Reducing the error in terrestrial laser scanning by optimizing the measurement set-up. In: XXI ISPRS Congress, Commission IVIII, 3-11 July 2008, Beijing, China, International Society for Photogrammetry and Remote Sensing.

Vanicek, P. and Krakiwsky, E. J., 2015. Geodesy: the concepts. Elsevier. 